\title{
Measuring surgical safety during minimally invasive surgical procedures: a validation study
}

\author{
Mathijs D. Blikkendaal ${ }^{1}$. Sara R. C. Driessen ${ }^{1}$. Sharon P. Rodrigues ${ }^{1}$. Johann P. T. Rhemrev ${ }^{2} \cdot$ Maddy J. G. H. Smeets $^{2}$. \\ Jenny Dankelman ${ }^{3} \cdot$ John J. van den Dobbelsteen ${ }^{3}$. Frank Willem Jansen ${ }^{1,3}$
}

Received: 22 July 2017 / Accepted: 3 January 2018 / Published online: 19 January 2018

(c) The Author(s) 2018. This article is an open access publication

\begin{abstract}
Background During the implementation of new interventions (i.e., surgical devices and technologies) in the operating room, surgical safety might be compromised. Current safety measures are insufficient in detecting safety hazards during this process. The aim of the study was to observe whether surgical teams are capable of measuring surgical safety, especially with regard to the introduction of new interventions.

Methods A Surgical Safety Questionnaire was developed that had to be filled out directly postoperative by three surgical team members. A potential safety concern was defined as at least one answer between (strongly) disagree and indifferent. The validity of the questionnaire was assessed by comparison with the results from video analysis. Two different observers annotated the presence and effect of surgical flow disturbances during 40 laparoscopic hysterectomies performed between November 2010 and April 2012.

Results The surgeon reported a potential safety concern in $16 \%$ (85/520 questions). With respect to the scrub nurse and anesthesiologist, this was both $9 \%(46 / 520)$. With respect to the preparation, functioning, and ease of use of the devices in $37.5-47.5 \%$ (15-19/40 procedures) a potential safety concern was reported by one or more team members. During procedures after which a potential safety concern was reported, surgical flow disturbances lasted a higher percentage of the procedure duration [9.3 \pm 6.2 vs. $2.9 \pm 3.7 \%$ (mean $\pm \mathrm{SD}$ ), $p<.001]$. After procedures during which a new instrument or device was used, more potential safety concerns were reported (51.2 vs. $23.1 \%, p<.001)$.

Conclusions Potential safety concerns were especially reported during procedures in which a relatively high percentage of the duration consisted of surgical flow disturbances and during procedures in which a new instrument or device was used. The Surgical Safety Questionnaire can act as a validated tool to evaluate and maintain surgical safety during minimally invasive procedures, especially during the introduction of a new intervention.
\end{abstract}

Keywords Interobserver reliability $\cdot$ Minimally invasive surgery $\cdot$ Video observation $\cdot$ Surgical safety

Electronic supplementary material The online version of this article (https://doi.org/10.1007/s00464-018-6021-7) contains supplementary material, which is available to authorized users.

Mathijs D. Blikkendaal

m.d.blikkendaal@lumc.nl

1 Department of Gynecology, Leiden University Medical Center, P.O. Box 9600, 2300 RC Leiden, The Netherlands

2 Department of Gynecology, Haaglanden Medical Center, P.O. Box 96900, 2509 JH The Hague, The Netherlands

3 Department of BioMechanical Engineering, Delft University of Technology, Mekelweg 2, 2628 CD Delft, The Netherlands
In the ongoing search for optimal patient outcomes, surgical procedures are continuously evolving [1]. As a result, maintaining the high level of patient safety has become a great challenge [2]. Implementing new techniques and/or technologies causes changes in standardized surgical procedures to which every surgical team member has to adapt [3, 4]. Monitoring surgical safety in the operating room (OR) is one of the most important issues to guarantee optimal surgical outcome. However, real-time monitoring of the surgical safety during a procedure is difficult. The question is: what and how should we monitor and who should do it?

Previous studies describing patient safety during minimally invasive surgery (MIS) have defined certain domains that are 'at risk' [5-8]. In daily practice the identification 
of these safety issues is often limited to observers that were physically present in the OR and retrospective interpretation of the obtained data $[6,9,10]$. Adequate interpretation is difficult and requires correct differentiation of errors (undesired actions) from events (consequence of undesired actions) [5]. Currently, patient safety indicators are frequently based on the occurrence of adverse events [11]. However, in general, intraoperative adverse events rarely occur. In theory, for an adverse event to occur several errors have to line up and slip through the holes of existing safety barriers [12]. Usually most errors that precede a potential adverse event are timely recognized and dealt with. However, these near-misses disturb the surgical flow to a greater or lesser extent and therefore interfere with surgical safety $[3-5,10,13-16]$.

In daily practice, there is no external observer present during a procedure. The only 'real-time monitoring' of patient safety is done by the surgeon and/or the entire surgical team itself. However, from a psychological perspective it is known that an individuals' situational awareness is impaired when occupied with a (difficult) task [17]. Regarding this phenomenon, implementing new surgical devices and technologies in the OR puts more pressure on the responsibility of the surgeon to maintain surgical safety during the whole procedure $[1,15]$. The only measures to enhance safety throughout a procedure that currently are-or at least should be-used, are the preoperative team briefings, the postoperative debriefings and, to a lesser extent, some preoperative checklists. In general, these safety instruments have proven to diminish preventable errors during the procedure and to safeguard open communication [18-21]. However, since these tools do not incorporate items to evaluate new surgical techniques or technologies, they are insufficient in detecting safety hazards during their introduction.

Therefore, the aim of this study was to observe whether surgical teams are capable of measuring surgical safety, especially with regard to the introduction of new techniques and technologies during a series of MIS procedures. A questionnaire that had to be filled out directly postoperative was developed to measure surgical safety. Next, the validity of the questionnaire was assessed by comparison with the results from independent video analysis of these procedures.

\section{Materials and methods}

In a university-affiliated teaching hospital (Haaglanden Medical Center, The Hague), a prospective registration study was set-up to record and analyze surgical flow disturbances. During a consecutive series of laparoscopic hysterectomies (LH), a questionnaire was filled out in the OR by the surgical team members. The surgical flow disturbances were scored by an independent observer. To minimize the interference of the study on its own results (the 'Hawthorne effect'), this observation was based on video registration of the procedures. Outcome measures were the number, types, effect, and duration of surgical flow disturbances per procedure.

The $\mathrm{LH}$ was chosen as procedure of interest, because it is an advanced laparoscopic procedure performed by a dedicated operating team and requiring a wide array of endoscopic instruments and equipment. The study started in November 2010 and all consecutive LHs that were performed in a conventional (cart-based) OR were registered until the start of the construction of the new integrated OR (Karl Storz OR1 ${ }^{\mathrm{TM}}$ integrated OR system, September 2011). After construction of the integrated OR (October 2011), the same amount of eligible procedures was registered in this setting. Similarly, the occasional introduction of new devices in both the conventional and integrated OR was registered. In this manner, not only the transition to the integrated OR, but also the introduction of new devices was analyzed. All procedures were performed by either of the two gynecologists with more than 10 years of experience in advanced gynecologic laparoscopy and were assisted by one gynecologist who conducted a fellowship in MIS; a group of five alternated in the position of either circulating or scrub nurse.

The study was approved by the Executive Board of the Haaglanden Medical Center. Prior to the start of the study, all OR personnel were collectively informed about the study. From each patient, informed consent was obtained. This design was adapted from another study [3].

\section{Development of Surgical Safety Questionnaire}

Patient safety risk factors that have been described by Rodrigues et al., were summarized in a questionnaire consisting of 13 questions (i.e., time-out/sign-out, preparation and functioning of devices and instruments, functioning of the surgical team, distracting stimuli, and interference of the study on the procedure) [6]. Directly after each procedure the (assisting-)surgeon, scrub nurse, and anesthetist(-assistant) filled out this short questionnaire. Answers were given on a 5-point Likert scale, ranging from (strongly) disagree to (strongly) agree. A potential safety concern was defined as an answer between (strongly) disagree and indifferent by at least one member of the surgical team. Additionally, several questions regarding experience (with the procedure, laparoscopy in general, and the used instruments/devices) and the procedure (adhesions, adverse events) were stated (see Online Appendix).

\section{Video analysis}

The input from three video signals (endoscopic image and two dome cameras) and four audio signals (MPEG Recorder 2.1) was synchronously recorded during all procedures. The recordings were started just before the time-out procedure 
and stopped after suturing all port-sites. The procedure was excluded from analysis in case of technical problems related to the recording equipment. Two residents in Obstetrics and Gynecology (M.D.B. and S.R.C.D.) analyzed the presence and effect of predefined surgical flow disturbances. These surgical flow disturbances were defined as stimuli distracting one or more members of the surgical team (Table 1). To assess the severity, the effect of the surgical flow disturbance on the surgical team members was graded according to a seven-point scale. This scale ranges from 1 as a potentially distracting stimulus to 7 when the sterile team's work is completely interrupted (modified by Persoon et al. originally described by Healey et al.) (Table 2) [9, 22].

Table 1 Observed types of surgical flow disturbances

\begin{tabular}{l}
\hline Equipment-/instrument-related \\
Set-up device/connection \\
Intraoperative repositioning \\
Malfunctioning \\
Not present \\
Sterility \\
Other/unclear \\
Environmental \\
Pager/telephone \\
Door washing room \\
Radio use \\
Personnel-related \\
Communication failure \\
Irrelevant conversation \\
Procedure-related \\
Extra coagulation bleeding-site \\
Unexpected adhesions \\
Limited vision (condensation/smoke) \\
Adverse event \\
Conversion to laparotomy \\
\hline
\end{tabular}

\section{Statistics}

To facilitate statistical analysis, the recordings were annotated with The Observer® XT 11.5 software (Noldus Information Technologies, Wageningen, The Netherlands). To assess the interobserver variability, a random sample of six recordings was scored by both observers. The findings of the two observers for these six procedures were compared and the interobserver agreement was calculated (compares events between two observations and takes the frequency and sequence into account; function incorporated in The Observer ${ }^{\circledR}$ XT 11.5 software). After satisfactory interobserver agreement was achieved, the remaining procedures were annotated by either one of the two observers (randomly allocated and analyzed in a non-chronological random order) [23, 24]. For statistical analysis, SPSS 23 statistical software was used. Intraclass correlation coefficient (ICC) was used to assess the inter-rater agreement. A two-way random effects model was used since both the procedures as well as the raters are a random sample from a larger pool of procedures and raters. We checked for consistency (i.e., raters have a similar pattern of scores). Outcomes are both average measures and single measures. Average measures provide the reliability of the score being able to separate different levels of safety, despite differences in individual scoring. Single Measures represent the reliability you would get if one rater was used. Values between 0.4 and 0.75 were considered to represent "fair to good reliability" and $>0.75$ "excellent reliability" [25]. In case the kappa becomes negative (due to low variability and high agreement), the absolute agreement was described as a percentage [26]. A Pearson Chi-square test was used to compare proportions and a Mann-Whitney $U$ test was used for continuous variables (non-normally distributed data). A $p<.05$ was considered statistically significant.

Table 2 Effect of observed surgical flow disturbances (according to Persoon et al. [9])

1 Events with the potential to distract the sterile team

2 Sterile team member momentarily distracted: possible involvement of a single sterile member in an event not related to the primary task, e.g., a short head turn in response to a visual or auditory stimulus

3 Sterile team member engages in distraction: similar distraction in 2, but the sterile member engages with the source of distraction by verbally responding while maintaining primary task activity (multitasking)

4 Sterile team member's primary task interrupted: a single team member ceases his/her current tasks to engage entirely in the distracting stimulus

5 Sterile team momentarily distracted: two or more sterile team members respond to a stimulus with a short head turn, no verbal response

6 Sterile team engage in secondary tasks: two or more team members engage with the source of distraction by verbally responding while maintaining primary task activity

7 Sterile team's work interrupted-operation flow disrupted: interruption of the current primary task of the sterile team, the operation flow is disrupted 


\section{Results}

During the study period, 84 LHs were performed of which 40 were eligible for inclusion in two studies [3]. For detailed information on the excluded procedures, see Fig. 1. All procedures were successfully completed and three minor postoperative complications were noted (Tables 3, 4).

The (assisting-)surgeon answered $95 \%$ of all questions [494 out of total 520 questions (40 procedures, 13 questions per procedure)], the scrub nurse answered $89 \%$ (461 out of 520), and the anesthetist(-assistant) answered $86 \%$ of the questions (445 out of 520). Based on the questionnaire, all surgical team members were of the opinion that the study did not interfere with the procedure in 33 out of the 40 procedures (83\%). In all cases, one of the two experienced gynecologists ( $>100 \mathrm{LHs}$ ) attended the procedure. Nevertheless, the questionnaire was filled out in 58\% of the cases by the assisting surgeon. As a result, reported experience of the surgeon with LH varied between $\leq 25$ prior procedures (14\%), 26-40 (30\%), 41-100 (32\%), and $>100$ prior LHs in $24 \%$ of the procedures. The surgeons reported in $41 \%$ of the cases to have used the same instruments and devices $>100$ times before in prior procedures. In $50 \%$ they reported to have experience with the equipment between 25 and 100 prior procedures and in $8 \%$ this was $\leq 25$ procedures. Experience of the scrub nurse with
Table 3 Patient and procedure characteristics of analyzed LHs performed in the Haaglanden Medical Center, The Hague, between January 2011 and April 2012

\begin{tabular}{llll}
\hline & \multicolumn{2}{l}{ Overall $(N=40)$} & Min-max \\
\cline { 2 - 4 } & Median & IQR & \\
\hline Age (years) & 48.2 & $43.9-55.2$ & \\
BMI $\left(\mathrm{kg} / \mathrm{m}^{2}\right)$ & 24.9 & $22.7-27.3$ & \\
Uterine weight $(\mathrm{g})$ & 165 & $97-256$ & \\
${\text { Operating time }(\mathrm{min})^{\mathrm{a}}}^{\mathrm{a}}$ & 121 & \pm 29 & $66-176$ \\
Procedure time (min) & 156 & \pm 31 & $98-215$ \\
Estimated blood loss (mL) $^{\mathrm{b}}$ & 100 & $50-175$ & \\
Hospital stay (days) & 2.0 & $1.1-2.1$ & \\
Benign indication $(\%)$ & $70.0 \%$ & & \\
\hline
\end{tabular}

$I Q R$ inter quartile range (25th and 75th percentile), BMI Body Mass Index

${ }^{\mathrm{a}}$ Time between first incision and last suture (skin-to-skin) (based on video observation)

${ }^{\mathrm{b}}$ Time between patient entering OR and leaving OR (based on video observation)

MIS was in $37 \%$ of the cases between 41 and 100 and in $53 \%>100$ prior procedures. Despite this, experience with LH specifically was moderate; in $71 \%$ of the cases the scrub nurse had performed $\leq 25$ prior LH procedures. Similarly, their experience with the equipment was moderate (in $43-47 \%$ of the cases $\leq 25$ procedures).

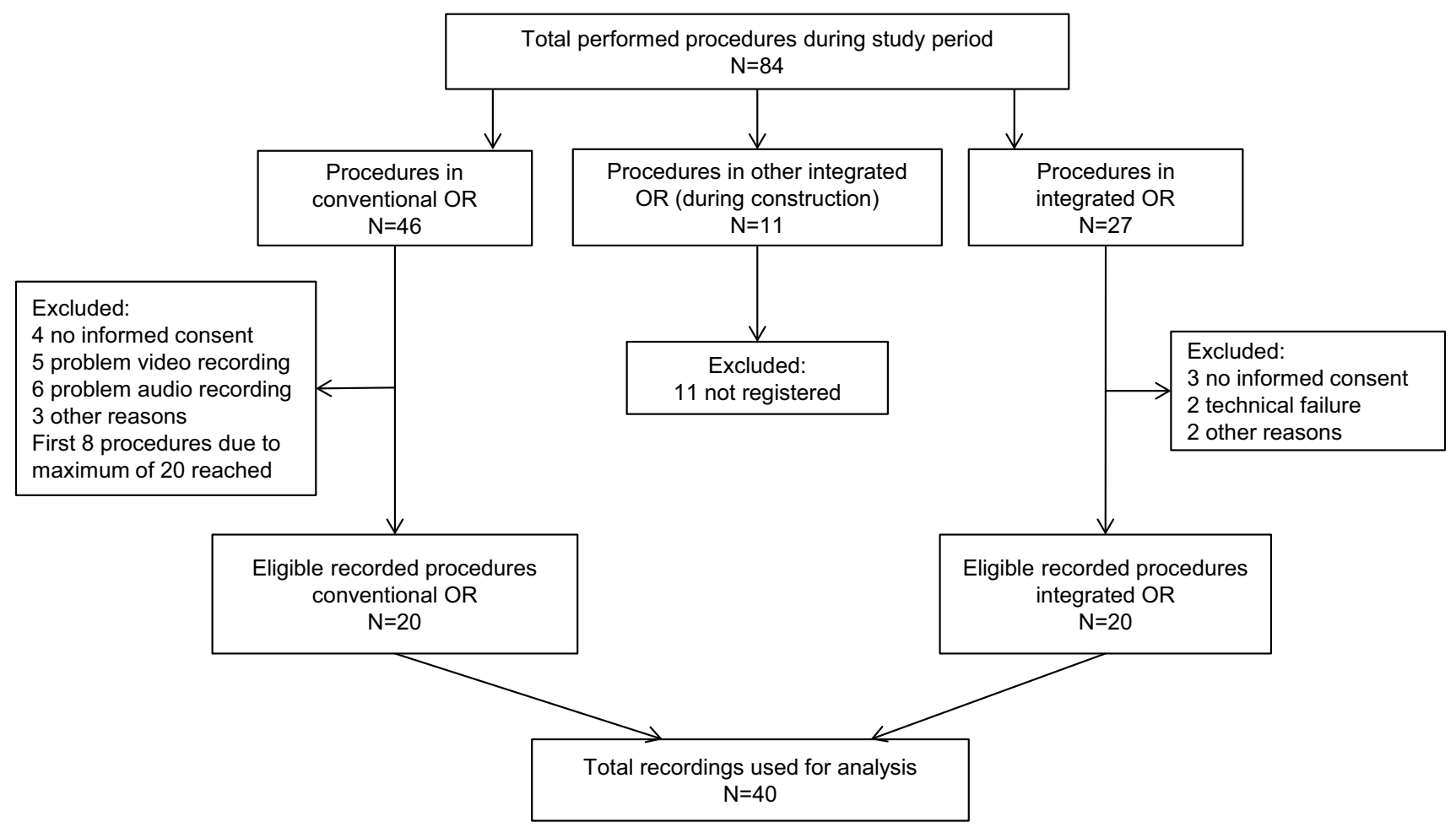

Fig. 1 Inclusion of eligible procedures 
Table 4 Adverse events all analyzed LHs

\begin{tabular}{ll}
\hline & Overall $(N=40)$ \\
\hline Infection & $1(2.5 \%)^{\mathrm{a}}$ \\
Blood loss $>1 \mathrm{~L}$ & $1(2.5 \%)^{\mathrm{b}}$ \\
Others & $1(2.5 \%)^{\mathrm{c}}$ \\
Total & $3(7.5 \%)$ \\
\hline
\end{tabular}

All adverse events did not require re-operation and occurred postoperatively

LH Laparoscopic hysterectomy

${ }^{\mathrm{a}}$ Urinary tract infection

${ }^{\text {b}}$ Postoperative drop in hemoglobin. CT-scan showed free fluid intraabdominally. Vital signs were stable and after a blood transfusion with two packed cells hemoglobin levels remained stable

${ }^{c}$ Patient suffered from sensibility loss in her right hand. The neurologist diagnosed a neurapraxia of the median nerve. Conservative management resulted in almost complete recovery

\section{Surgical Safety Questionnaire}

The scores per question of the individual team members are summarized in Table 5. In 15\% (6 out of 40) of the procedures, potential safety concerns [i.e., answer 'indifferent' or '(strongly) disagree'] were reported regarding the time-out and sign-out procedure. With respect to the preparation, functioning, and ease of use of the devices in $37.5-47.5 \%$ (15-19 out of 40 procedures) a potential safety concern was reported by one or more team members. A strong disagreement to a flawless use of the devices was reported in seven procedures (17.5\%). With respect to communication and collaboration in 30-35\% (12-14 out of 40 procedures) concerns were reported, mostly by the surgeon.
In general, scores given by the surgeon were in $16 \%$ $(85 / 520)$ regarded as a potential safety concern. With respect to the scrub nurse and anesthesiologist this was both $9 \%$ (46/520). Overall, 'strongly disagree' was reported in $2 \%$ $(9 / 520)$, of which 8 were reported on questions 3 , 4, or 5 (i.e., equipment related, see Online Appendix).

In $87 \%$ (452 of 520 questions), all members of the surgical team agreed in their answers (i.e., the maximum difference between the lowest and the highest was $\leq$ one point on the Likert scale). In $4 \%$ (22 of 520), the absolute difference between the members of the surgical team was high ( $\geq 3$; for example, to the same question the surgeon reports 'disagree' and the scrub nurse reports 'strongly agree'). The ICC was 0.44 (average measures).

\section{Validation of Surgical Safety Questionnaire by video analysis}

The overall observation duration of these procedures was $103 \mathrm{~h}$ and $45 \mathrm{~min}$. Six randomly chosen observations were annotated by both observers and showed excellent agreement (Cohen's Kappa of 0.79-0.98, all observations combined $0.85, p<.001$ ). Therefore, the remaining procedures were annotated by the two observers separately (in total 36 observations by M.D.B. and 10 by S.R.C.D., respectively). The duration and effect of disturbances during procedures in which a potential safety concern was reported with regard to the functioning of devices and instruments (question 4, see Online Appendix) were compared to the procedures in which no safety concern was reported (Table 6). In the procedures after which a potential safety concern was reported, a significantly higher percentage of the duration of the procedure consisted of surgical flow disturbances $[9.3 \pm 6.2$ vs.

Table 5 scores per question of the team members individually

\begin{tabular}{|c|c|c|c|c|c|c|c|c|c|c|c|c|}
\hline \multirow[t]{2}{*}{ Question } & \multicolumn{4}{|c|}{ Surgeon } & \multicolumn{4}{|c|}{ Scrub nurse } & \multicolumn{4}{|c|}{ Anesthetist } \\
\hline & $N$ & Mean \pm SD & Range & $N \leq 3$ & $N$ & Mean \pm SD & Range & $N \leq 3$ & $N$ & Mean \pm SD & Range & $N \leq 3$ \\
\hline Time-out & 39 & $4.54 \pm 0.55$ & $3-5$ & 1 & 36 & $4.19 \pm 0.67$ & $2-5$ & 3 & 37 & $4.08 \pm 0.68$ & $2-5$ & 3 \\
\hline Sign-out & 37 & $4.49 \pm 0.51$ & $4-5$ & 0 & 31 & $4.16 \pm 0.86$ & $2-5$ & 5 & 28 & $3.96 \pm 0.51$ & $2-5$ & 2 \\
\hline Preparation & 39 & $3.97 \pm 1.06$ & $1-5$ & 11 & 36 & $4.14 \pm 0.72$ & $2-5$ & 5 & 34 & $3.88 \pm 0.81$ & $2-5$ & 7 \\
\hline Functioning & 39 & $3.51 \pm 1.21$ & $1-5$ & 16 & 36 & $3.83 \pm 1.11$ & $1-5$ & 6 & 33 & $3.85 \pm 0.67$ & $2-5$ & 6 \\
\hline Ease of use & 39 & $3.82 \pm 1.07$ & $1-5$ & 11 & 36 & $3.94 \pm 0.83$ & $2-5$ & 5 & 32 & $3.81 \pm 0.74$ & $1-5$ & 7 \\
\hline Communication & 39 & $3.9 \pm 0.75$ & $2-5$ & 11 & 35 & $3.86 \pm 0.77$ & $2-5$ & 5 & 36 & $4.11 \pm 0.52$ & $3-5$ & 3 \\
\hline Collaboration & 39 & $3.92 \pm 0.74$ & $2-5$ & 10 & 36 & $3.89 \pm 0.62$ & $2-5$ & 5 & 36 & $4.14 \pm 0.42$ & $3-5$ & 1 \\
\hline Disturbances & 39 & $3.95 \pm 0.92$ & $2-5$ & 7 & 36 & $3.89 \pm 0.85$ & $1-5$ & 4 & 35 & $3.77 \pm 0.81$ & $2-5$ & 8 \\
\hline Surgeon & 28 & $3.96 \pm 0.43$ & $3-5$ & 3 & 36 & $4.25 \pm 0.55$ & $3-5$ & 2 & 35 & $4.14 \pm 0.49$ & $3-5$ & 2 \\
\hline Scrub nurse & 39 & $3.92 \pm 0.62$ & $2-5$ & 7 & 35 & $4 \pm 0.48$ & $3-5$ & 4 & 35 & $4.14 \pm 0.43$ & $3-5$ & 1 \\
\hline Anesthetist & 39 & $4.18 \pm 0.51$ & $3-5$ & 2 & 36 & $4.19 \pm 0.47$ & $3-5$ & 1 & 32 & $4.41 \pm 0.5$ & $4-5$ & 0 \\
\hline Patient safety & 39 & $4.21 \pm 0.7$ & $3-5$ & 4 & 36 & $4.08 \pm 0.5$ & $2-5$ & 1 & 36 & $4.42 \pm 0.5$ & $4-5$ & 0 \\
\hline Study influence & 39 & $4.56 \pm 0.6$ & $3-5$ & 2 & 36 & $4.31 \pm 0.47$ & $4-5$ & 0 & 36 & $3.97 \pm 0.81$ & $2-5$ & 6 \\
\hline
\end{tabular}

$N \leq 3$ : The number of questions to which a score $\leq 3$ was given, which is defined as a safety concern 
Table 6 Duration and effect of surgical flow disturbances with regard to functioning of devices and instruments (question 4 of questionnaire) separated between procedures with or without a safety concern reported by at least one member of the surgical team $(N=40$ procedures)

\begin{tabular}{|c|c|c|c|c|c|c|c|}
\hline & \multicolumn{3}{|c|}{ No safety concern reported ${ }^{\mathrm{d}}$} & \multicolumn{3}{|c|}{ Safety concern reported ${ }^{\mathrm{d}}$} & \multirow[b]{2}{*}{$p_{\mathrm{e}}$} \\
\hline & $N$ & Mean \pm SD & Min-max & $N$ & Mean \pm SD & Min-max & \\
\hline Percentage of procedure ${ }^{a}$ & 21 & $2.9 \pm 3.7$ & $0.0-15.4$ & 19 & $9.3 \pm 6.2$ & $1.6-21.7$ & $<.001$ \\
\hline Effect (weighted) ${ }^{b}$ & 21 & $4.4 \pm 2.4$ & $0.0-7.0$ & 19 & $6.1 \pm 1.9$ & $3.0-7.0$ & .020 \\
\hline Impact $^{\mathrm{c}}$ & 21 & $13.2 \pm 12.0$ & $0.0-47.1$ & 19 & $56.2 \pm 38.7$ & $11.5-145.7$ & $<.001$ \\
\hline
\end{tabular}

$S D$ standard deviation

${ }^{a}$ Total duration of the disturbance (s) defined as percentage of the total procedure time

${ }^{b}$ Effect of the disturbance (based on Persoon et al. [9]) corrected by the duration of the disturbance(s)

${ }^{c}$ Percentage of procedure multiplied by weighted effect

${ }^{\mathrm{d}}$ Reported answer by at least one surgical team member was (strongly) disagree or indifferent

${ }^{\mathrm{e}}$ Mann-Whitney U test for independent samples

$2.9 \pm 3.7 \%($ mean $\pm \mathrm{SD}), p<.001]$. Similarly, in these procedures, a significantly higher mean weighted effect (i.e., the mean effect of the disturbances corrected for the duration of the disturbances) was found (score $6.1 \pm 1.9$ vs. $4.4 \pm 2.4$, $p=.020$; see Table 2 for the meaning of the scores).

In the group without any reported safety concerns, there were only two procedures during which a relatively high percentage of the procedure consisted of disturbances (10.0 and $15.4 \%$, respectively). However, the mean weighted effect of these disturbances was low (1.9 and 3.0, respectively) and therefore can be regarded as adequately managed. All tests to assess whether using the questionnaire of one or two of the team members might be applicable as well resulted in lower agreement with the video analysis (not shown).

\section{Newly introduced devices and/or technology}

During eight procedures (20\%, four procedures in the conventional OR and four in the integrated OR), a new instrument and/or device was used. During these procedures, the surgical team members reported a potential safety concern in $51 \%$ (41 out of 80 questions regarding intraoperative aspects (question 3 till 12), see Online Appendix). In contrast, the prevalence of a potential safety concern during the other procedures was $23.1 \%$ (74 out of $320, p<.001$ ).

The first 20 procedures were performed in a conventional cart-based OR. The last 20 procedures were performed in a new integrated OR. No difference in potential safety concerns was reported between the two OR set-ups (28 vs. 29\%, $p=.740$ ). Furthermore, an employee of the medical industry was present during seven procedures (four in conventional OR, three in integrated OR), during which a newly introduced device was used. Additionally, in one procedure a new device was used without an employee of the industry being present (fourth consecutive procedure in which this instrument was used). The new equipment concerned a new bipolar sealing instrument (five procedures), a new type of suture for the vaginal cuff (one procedure), and multiple new devices/instruments (three procedures).

\section{Experience}

Limited experience of the scrub nurse with the equipment ( $\leq 25$ procedures) resulted in significantly more potential safety concerns reported by at least one member of the surgical team (30.7 vs. $15.6 \%, p=.002)$. However, this did not result in a higher percentage of procedure time expended to surgical flow disturbances $(7.3 \pm 7.6$ vs. $5.0 \pm 5.2 \%, p=.423)$ and/or a higher effect of these disturbances $(5.7 \pm 1.4$ vs. $4.8 \pm 2.3, p=.275)(N=30$ procedures $)$. Experience of the surgeon with the used instruments did not have a significant influence on the potential safety concerns either (25.6 vs. $23.8 \%, p=.791)$.

\section{Discussion}

The Surgical Safety Questionnaire filled out directly postoperative by all members of the surgical team proved to be a valid tool to adequately estimate surgical safety in MIS. Procedures during which a relatively high percentage of the duration consisted of surgical flow disturbances and/or with a relatively high mean weighted effect of these disturbances matched with the reported potential safety concerns. Furthermore, during procedures in which a new instrument or device was used, significantly more potential safety concerns were reported by the surgical team. Therefore this could be a useful tool in the evaluation and maintenance of surgical safety during the introduction of new surgical equipment or technology.

The term patient safety is at risk to become an empty phrase by its broad interpretation. To define nuances in patient safety, the 'systems approach' is most commonly used [27, 28]. Based on this approach, several studies 
introduced frameworks covering the risk domains relevant to surgical safety and patient outcomes $[6,7,29]$. The questionnaire validated in present study covers these risk domains and thereby provides a composite outcome for surgical safety.

A study conducted by Russ et al. had similar objectives and described the Metric for Evaluating Task Execution in the Operating Room (METEOR) as an easy to use tool to allow surgical teams to self-assess their performance, in order to track surgical hazards, and to be able to evaluate safety [30]. However, their checklist is quite extensive (up to 80 items) and does not cover concerns regarding instruments and devices. Since the high dependency on technology in MIS, equipment-related disturbances are one of the well-known primary sources of disruption [3, 8, 31]. Additionally, during the introduction of a new technique and/or technology in the OR, disruptions are even more likely to occur $[4,7]$. This hazard is also one of the main results in our study. Therefore, prior to the introduction of a new intervention in the $\mathrm{OR}$, a prospective risk analysis should be performed to guarantee safe implementation (e.g., Healthcare Failure Mode and Effect Analysis) [32]. Nevertheless, in our opinion, methods currently used to monitor this implementation (i.e., evaluation after 6 and 12 months, adverse events registration, incident reporting system) fail to detect safety concerns in a timely manner. Similarly, our results rule out the widespread assumption that an employee of the medical industry being present can prevent safety hazards. Instead, the Surgical Safety Questionnaire presented in this study could be a useful tool to systematically evaluate the surgical safety after each procedure, especially in case of the introduction of a new instrument or technology.

The main strength of our study is that by using video observation we were able to assess surgical flow disturbances without influencing the course of the procedure. In that way, we obtained very reliable quantitative results to serve as gold standard and thereby allowing validation of our Surgical Safety Questionnaire. This is in line with other studies recognizing the additional value of detailed analysis of video registration [33, 34]. A weakness could be that scoring on a 5-point Likert scale remains prone to subjectivity. What determines the difference between agree, neither agree nor disagree, and disagreement? It was decided to place the cut-off for a potential safety concern at 'neither agree nor disagree.' By doing so, every time at least one of the team members for any reason had a motive to not (fully) agree on a certain question in the questionnaire, the item was marked as potential safety concern. Nevertheless, the results of our study indicate that by using this definition the potential safety concerns correlate very well with the observed surgical flow disturbances.
Furthermore, in contrast to the high agreement (87\%), the reported ICC (0.44) seems low. However, this discrepancy is explained by the low variability and high agreement in the reported answers. In those cases, kappa is not a reliable estimate for correlation [26]. Thirdly, the reported experience with the LH seems low. This is due to the system in The Netherlands, in which residents specializing in MIS are usually allowed to perform LH as 'primary' surgeon during the last year of their residency and therefore also filled out our scoring sheets. However, without exception, in these cases, the senior consultant with extensive experience in advanced gynecologic endoscopy was always member of the sterile team as well.

Over the past decades patient outcomes regarding MIS have rapidly improved. Large leaps could be made in the early days of MIS, where measures taken to improve safety were highly effective. Currently, only smaller steps can be made with a higher risk of doing harm instead of good [1, 35]. Furthermore, the OR has become increasingly complex. As Sir Cyril Chantler said: "Medicine used to be simple, ineffective and relatively safe. Now it is complex, effective and potentially dangerous" [36]. The common objective we are pursuing is to enable technology to assist the surgeon and its team in maintaining surgical safety. Similar to recent developments in the automotive industry to assist the driver on traffic safety (e.g., collision avoidance, blind spot detection, and lane departure warning systems), some promising systems are currently tested in a few hospitals in The Netherlands. For example, the Digital Operating Room Assistant continuously monitors the location, status, and (mal)functioning of devices [37, 38].

In conclusion, the results of our study demonstrate that the presented Surgical Safety Questionnaire can act as a validated tool to evaluate and maintain surgical safety during minimally invasive procedures. In daily practice, we recommend to fill out this questionnaire in case a new technique or technology is used during a procedure. By involving the complete surgical team with their individual knowledge, experience, and opinions, this will provide the opportunity to constantly evaluate new equipment and techniques. As a consequence, in an early stage, potential safety hazards will be prevented in future patients.

Acknowledgements The authors want to acknowledge Arjan van Dijke for his extensive help with the video observation system.

\section{Compliance with ethical standards}

Disclosures Mathijs Blikkendaal, Sara Driessen, Sharon Rodrigues, Johann Rhemrev, Maddy Smeets, Jenny Dankelman, John Dobbelsteen, and Frank Willem Jansen have no conflicts of interest or financial ties to disclose. 
Open Access This article is distributed under the terms of the Creative Commons Attribution 4.0 International License (http://creativecommons.org/licenses/by/4.0/), which permits unrestricted use, distribution, and reproduction in any medium, provided you give appropriate credit to the original author(s) and the source, provide a link to the Creative Commons license, and indicate if changes were made.

\section{References}

1. Eisenberg D, Wren SM (2014) Innovation in safety, and safety in innovation. JAMA Surg 149:7-9

2. Sevdalis N, Hull L, Birnbach DJ (2012) Improving patient safety in the operating theatre and perioperative care: obstacles, interventions, and priorities for accelerating progress. $\mathrm{Br} \mathrm{J}$ Anaesth 109(Suppl 1):i3-i16

3. Blikkendaal MD, Driessen SR, Rodrigues SP, Rhemrev JP, Smeets MJ, Dankelman J, van den Dobbelsteen JJ, Jansen FW (2017) Surgical flow disturbances in dedicated minimally invasive surgery suites: an observational study to assess its supposed superiority over conventional suites. Surg Endosc 31:288-298

4. Antoniadis S, Passauer-Baierl S, Baschnegger H, Weigl M (2014) Identification and interference of intraoperative distractions and interruptions in operating rooms. J Surg Res 188:21-29

5. Bonrath EM, Dedy NJ, Zevin B, Grantcharov TP (2013) Defining technical errors in laparoscopic surgery: a systematic review. Surg Endosc 27:2678-2691

6. Rodrigues SP, Wever AM, Dankelman J, Jansen FW (2012) Risk factors in patient safety: minimally invasive surgery versus conventional surgery. Surg Endosc 26:350-356

7. Driessen SRC, Sandberg EM, Rodrigues SP, van Zwet EW, Jansen FW (2017) Identification of risk factors in minimally invasive surgery: a prospective multicenter study. Surg Endosc 31:2467-2473

8. Weerakkody RA, Cheshire NJ, Riga C, Lear R, Hamady MS, Moorthy K, Darzi AW, Vincent C, Bicknell CD (2013) Surgical technology and operating-room safety failures: a systematic review of quantitative studies. BMJ Qual Saf 22:710-718

9. Persoon MC, Broos HJ, Witjes JA, Hendrikx AJ, Scherpbier AJ (2011) The effect of distractions in the operating room during endourological procedures. Surg Endosc 25:437-443

10. Parker SE, Laviana AA, Wadhera RK, Wiegmann DA, Sundt TM III (2010) Development and evaluation of an observational tool for assessing surgical flow disruptions and their impact on surgical performance. World J Surg 34:353-361

11. Pronovost PJ, Thompson DA, Holzmueller CG, Lubomski LH, Morlock LL (2005) Defining and measuring patient safety. Crit Care Clin 21:1-19

12. Reason J (2004) Beyond the organisational accident: the need for "error wisdom" on the frontline. Qual Saf Health Care 13(Suppl 2):ii28-ii33

13. Wiegmann DA, ElBardissi AW, Dearani JA, Daly RC, Sundt TM III (2007) Disruptions in surgical flow and their relationship to surgical errors: an exploratory investigation. Surgery 142:658-665

14. Weigl M, Antoniadis S, Chiapponi C, Bruns C, Sevdalis N (2015) The impact of intra-operative interruptions on surgeons' perceived workload: an observational study in elective general and orthopedic surgery. Surg Endosc 29:145-153

15. Pluyter JR, Buzink SN, Rutkowski AF, Jakimowicz JJ (2010) Do absorption and realistic distraction influence performance of component task surgical procedure? Surg Endosc 24:902-907

16. Hsu KE, Man FY, Gizicki RA, Feldman LS, Fried GM (2008) Experienced surgeons can do more than one thing at a time: effect of distraction on performance of a simple laparoscopic and cognitive task by experienced and novice surgeons. Surg Endosc 22:196-201
17. Johnson HL, Kimsey D (2012) Patient safety: break the silence. AORN J 95:591-601

18. Treadwell JR, Lucas S, Tsou AY (2014) Surgical checklists: a systematic review of impacts and implementation. BMJ Qual Saf 23:299-318

19. Gluck PA (2012) Patient safety: some progress and many challenges. Obstet Gynecol 120:1149-1159

20. de Vries EN, Prins HA, Crolla RM, den Outer AJ, van Andel G, van Helden SH, Schlack WS, van Putten MA, Gouma DJ, Dijkgraaf MG, Smorenburg SM, Boermeester MA (2010) Effect of a comprehensive surgical safety system on patient outcomes. New Engl J Med 363:1928-1937

21. Birkmeyer JD (2012) Progress and challenges in improving surgical outcomes. Br J Surg 99:1467-1469

22. Healey AN, Primus CP, Koutantji M (2007) Quantifying distraction and interruption in urological surgery. Qual Saf Health Care 16:135-139

23. Buzink SN, van LL, de Hingh, Jakimowicz IH JJ (2010) Risksensitive events during laparoscopic cholecystectomy: the influence of the integrated operating room and a preoperative checklist tool. Surg Endosc 24:1990-1995

24. Zheng B, Martinec DV, Cassera MA, Swanstrom LL (2008) A quantitative study of disruption in the operating room during laparoscopic antireflux surgery. Surg Endosc 22:2171-2177

25. Fleiss JL (1986) The design and analysis of clinical experiments. Wiley, New York

26. Feinstein AR, Cicchetti DV (1990) High agreement but low kappa: I. The problems of two paradoxes. J Clin Epidemiol 43:543-549

27. Vincent C, Moorthy K, Sarker SK, Chang A, Darzi AW (2004) Systems approaches to surgical quality and safety: from concept to measurement. Ann Surg 239:475-482

28. Dankelman J, Grimbergen CA (2005) Systems approach to reduce errors in surgery. Surg Endosc 19:1017-1021

29. Calland JF, Guerlain S, Adams RB, Tribble CG, Foley E, Chekan EG (2002) A systems approach to surgical safety. Surg Endosc 16:1005-1014

30. Russ S, Arora S, Wharton R, Wheelock A, Hull L, Sharma E, Darzi A, Vincent C, Sevdalis N (2013) Measuring safety and efficiency in the operating room: development and validation of a metric for evaluating task execution in the operating room. J Am Coll Surg 216:472-481

31. Verdaasdonk EG, Stassen LP, van der Elst M, Karsten TM, Dankelman J (2007) Problems with technical equipment during laparoscopic surgery. An observational study. Surg Endosc 21:275-279

32. Linkin DR, Sausman C, Santos L, Lyons C, Fox C, Aumiller L, Esterhai J, Pittman B, Lautenbach E (2005) Applicability of healthcare failure mode and effects analysis to healthcare epidemiology: evaluation of the sterilization and use of surgical instruments. Clin Infect Dis 41:1014-1019

33. Bezemer J, Cope A, Korkiakangas T, Kress G, Murtagh G, Weldon SM, Kneebone R (2017) Microanalysis of video from the operating room: an underused approach to patient safety research. BMJ Qual Saf 26:583-587

34. Makary MA (2013) The power of video recording: taking quality to the next level. JAMA 309:1591-1592

35. Parsons JK, Messer K, Palazzi K, Stroup SP, Chang D (2014) Diffusion of surgical innovations, patient safety, and minimally invasive radical prostatectomy. JAMA Surg 149:845-851

36. Chantler C (1999) The role and education of doctors in the delivery of health care. Lancet 353:1178-1181

37. Guedon AC, Wauben LS, Overvelde M, Blok JH, van der Elst M, Dankelman J, van den Dobbelsteen JJ (2014) Safety status system for operating room devices. Technol Health Care 22:795-803 
38. Henken KR, Jansen FW, Klein J, Stassen LP, Dankelman J, van den Dobbelsteen JJ (2012) Implications of the law on video recording in clinical practice. Surg Endosc 26:2909-2916 